\title{
Incidence and Variations of Aberrant Renal Arteries in Kidney Donors Cases
}

\author{
Mustafa AbdulMonamZainel ${ }^{1}$, Suhelmawloodalnajjar ${ }^{1}$ \\ ${ }^{1}$ Department of Anatomy, Hawler Medical University, College of Medicine. Iraq, Irbil
}

\begin{abstract}
The origin of renal arteries, vessel diameter, and point of entry to the kidney has all been analyzed as potential parameters which are a clinically relevant issue. This anatomical feature may be essential for fully understanding the process of renal perfusion, as it is not only the number of renal arteries that influences the extent of renal blood supply, also their diameter. The aberrant renal arteries that supply the inferior pole are more important because it provides arterial supply to the proximal part of the ureter. The objective of this study is to identify influences and prevalence of aberrant renal arteries of the kidney donors' cases to perform role of the aberrant renal artery.

Prevalence of aberrant renal artery was identified in $26.3 \%$ of the cases, incidence was more frequent in left than right side; about $78.7 \%$ of cases presented unilaterally. The remaining cases $21.2 \%$ were RAs presented bilaterally. Diameter of main RA in kidney presenting the RA was $5.73 \mathrm{~mm}$ and that of kidney with only single renal artery was $6.19 \mathrm{~mm}$.
\end{abstract}

Keywords: Aberrant renal artery, renal arterial variations, renal angiography, kidney, incidence.

\section{Introduction}

As a wide range of renal vascular variations has been reported by anatomists and radiologists; prevalence of these variations is extremely divergent in various populations. Historically, first recorded aberrant renal artery (aRA) was reported by Eustachius ${ }^{[1]}$ in one of his famous plates which remained unprinted in Papal Library until 1714. Since then various cases of aRA have been reported; including variations in origin, number, and branching pattern of renal arteries [2\&3]. Therefore, as much as larger samples studied will give more clarifications and changes ${ }^{[3]}$.

aRA defined by presence of more than one renal artery (RA) supplying kidney which may arise from

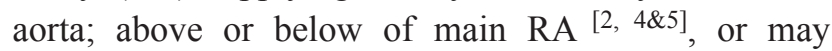
arise from coeliac, superior mesenteric [2], inferior mesenteric, and common iliac arteries ${ }^{[2 \& 3]}$. name "aberrant" described in literature whereby additional artery branches from abdominal aorta either superior or inferior to main RA and enters kidney directly through the capsule at the upper or lower poles of the kidney, or as "accessory" whereby it enters the hilum directly. Anatomical variants in the renal vasculature considered being extremely common variants ${ }^{[1,6 \& 7]}$; the prevalence of aRAs are found in 30\% (ranging 20-30\%) of the individuals ${ }^{[2-5]}$.

Embryologically, as the kidneys ascend from the pelvis during the embryological development, they receive their blood supply initially from common iliac arteries. Later, they receive new branches from the aorta, and the inferior branches disappear. This continuous generation and degeneration of renal arteries as the kidney ascend explains the high incidence of the variations in the blood supply to the kidneys. Therefore, the complicated embryology variations of this organ are relatively higher when compared with the others ${ }^{[8 \& 9]}$.

The main objectives and aims identify the prevalence and variation of the aberrant renal arteries of the kidney donors' cases, as they had been regarded as perfect normal cases for the study. Determine the anatomy and topography of the aberrant artery and main renal artery donor kidneys and preserving total donated kidney function.

Material and methods This study was designed to determine the main RAs and aRAs including its morphometric and location on each side in kidney 
donors. A double-blinded prospective and retrospective angiographic and morphometric imaging study performed on one hundred and eighty-four (184) potential prospective living kidney donors. This study was conducted from December 2018 to May 2019 at the radiology and imaging centre in Zheen International Hospital in Erbil city, Iraq. Only the cases that fit for the donation were selected medico-legally. This study was approved by the research ethics committee of the college of medicine, and written consent was received from the cases. Regarding the contrast media allergy cases, no hypersensitivity cases were recorded, other than some simple cutaneous rashes in two cases which need no interferences.

Computed Tomographic Angiographic (CTAngiographic) imaging for the cases performed according to the four-phase protocol; which include an initial non-contrast image acquisitionphase, followed by arterial phase, nephrographic phase, and excretory phase (delayed phase). The perfect anatomic coverage for CT-Angiography was extended between the dome of the diaphragm and the distal portion of the common iliac arteries or pelvic region. The dynamic CT-Angiography examination was performed with a 128-slice CT scanner (Somatom Definition AS+ 2012, SIEMENS Healthcare, and Germany) such that four phases were covered.

Finally, all images were reconstructed andanalyzed by Syngo program (Siemens Healthcare, Germany). In 3D CT-Angiography, the VRT, MRP and MIP images were used for evaluation of the renal arteries. Regarding the RAs morphometry (diameter); retrospective analysis of the RAs of all the kidney donors was carried out in a double blinded fashion and measured at the aortic origin. To carry out the analysis of renal arteries variations and diameter based on the type of arterial blood supply present, the total number of the kidneys and arteries were established and then divided into different groups according to the following criteria; Gender, Side, Anatomic eRA - early branch renal artery, a single renal artery originating from the aorta and giving branch before reaching renal hilum and supplying poles, hilum of the kidney, and extra renal organs.

\section{Statistical Analysis}

Statistical analysis of the current study data was done by using social package for scientific statistic (SPSS) software version 24 (IBM Corporation, Armonk, New York, USA).
Result: The CT-Angiographic findings analyzed in one hundred and eighty-four (184) cases i.e. three hundred and sixty-eight (368) bilateral kidneys were enrolled in this study. Cases are distributed between 128 males and 56 females. Participants' age ranged between 19-61 years for males and 19-58 years for females. The mean age of males is $(24.05 \pm 6.4)$ years, and the females mean age is $(28.12 \pm 7.9)$ years; which there are no statistically significant differences between these two groups (t-test, $\mathrm{P}=0.961$ ).

The incidence of only single RA is identified in 271 (73.64\%) out of 368 kidneys. Whereas the renal arterial variations i.e. aRA were identified in 97 (26.3\%) out of 368 kidneys.

Accordingly, the incidence of aRA on the right side is $38(20.7 \%)$ out of 184 kidneys. While on the left side, the incidence is $59(32.1 \%)$ out of 184 kidneys. There are statistically significant differences between the left and right side, as the incidence of aRAs were more frequent in the left than the right side (Chi-square test, $\mathrm{P}=0.001$ )

The distribution of RAs that are supplying the right kidney classified as follow:

a) Only one

b) single renal artery is identified in $149(79.3 \%)$ kidneys.

c) Double renal arteries (one main RA and one aRA) were identified in 37 (20.1\%) kidneys.

d) Triple renal arteries (one main RA and two aRA) were identified in $1(0.5 \%)$ kidney which considered one of the rare cases.

Regarding the left side, number of RAs that supplying the kidney distributed as follow:

a) One single renal artery was identified in 125 (67.9\%) kidneys.

b) Double renal arteries (one main RA and one aRA) were identified in 53 (28.8\%) kidneys.

c) Triple renal arteries (one main RA and two aRA) were identified in 5 (2.7\%) kidneys.

Importantly, one rare case recorded in this study in which the left kidney supplied by quaternary renal arteries (one main RA and three aRA) were identified in $1(0.5 \%)$ kidney. Therefore, a statistically significant 
difference between the left and right side, as the incidence of multiple RAs were more frequent in the left than the right side (Chi-square test, $\mathrm{P}=0.001$ ). demonstrating the RA and aRAs. Regarding the prevalence of the renal arteries among genders. Although there were large differences in aRAs frequency between males and females (more frequent in males) in the current study but these differences were not statistically significant (Chisquare, $\mathrm{P}=0.663$ )

Among the 80 cases with aRAs, 63 (78.7\%) cases presented unilaterally. The remaining cases 17 (21.2 $\%)$ were the aRAs presented bilaterally. There is a statistically significant difference between groups with respect to the symmetricity were unilateral incidence was higher than bilateral incidence (Chi-square test, $\mathrm{P}<$ 0.001).
Regarding the origin of renal arteries, the total arteries that are originating from the aorta and other sources supplying the right kidney are 223 renal arteries distributed as 184 of single main RAs and 39 aRAs. In the left side, the total number of the renal arteries were studied are 249 renal arteries originating from the aorta and other sources distributed as 184 of single main RAs and 65 aRAs. Regarding the rare cases, only one $(2.5$ $\%)$ aRA was originated from the superior mesenteric artery was identified in the right side. In the left side, only one $(1.5 \%)$ aRA was identified to originate from the left common iliac artery. Table 1 summarizing the origin of renal arteries from the aorta and other sources.

Table 1: Distribution of renal arteries origin.

\begin{tabular}{|c|c|c|c|c|}
\hline & Single RA (n.) & $\mathbf{( \% )}$ & aRAs (n.) & (\%) \\
\hline Right side origin & & & & \\
\hline Aorta & 184 & 100 & 38 & 97.4 \\
\hline Superior mesentericartery & 0 & 0 & 1 & 2.5 \\
\hline Right common iliac artery & 0 & 0 & 0 & 0 \\
\hline Total & 184 & 100 & 39 & 100 \\
\hline Left side origin & & & & \\
\hline Aorta & 184 & 100 & 62 & 95.3 \\
\hline Trunk of inferior mesenteric artery & 0 & 0 & 2 & 3 \\
\hline Superior mesenteric artery & 0 & 0 & 0 & 0 \\
\hline Left common iliac artery & 0 & 0 & 1 & 1.5 \\
\hline Total & 184 & 100 & 65 & 100 \\
\hline
\end{tabular}
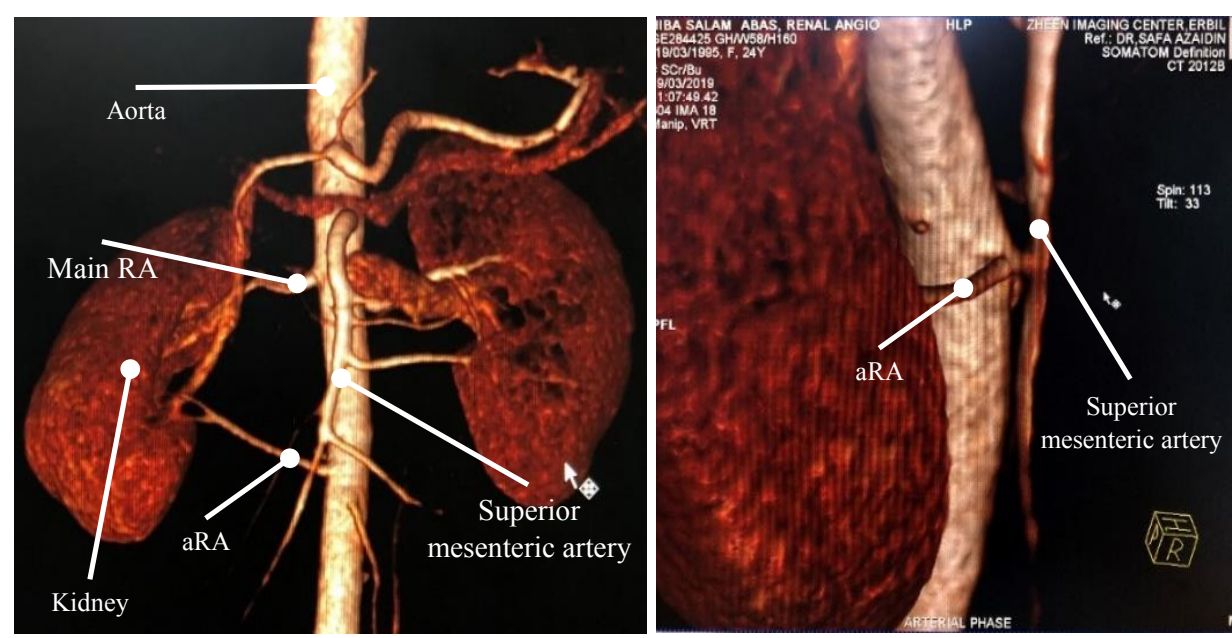

Figure 1: One of the rare cases demonstrating the aRA arising from superior mesenteric artery and merge with aorta and forming one artery supplying the lower pole of the right kidney. 
The entrance of the main renal artery and aberrant renal arteries to the kidney:

The RAs that's entering the kidney either to the kidney hilum or lower and upper poles calculated based on the total number of RAs supplying kidneys. In the right side, the number of arteries supplying the kidney is
223 RAs. All the single main RAs 184 (100\%) was found to enter the kidney through the hilum. In the left kidney, the number of arteries supplying kidney is 249 RAs. All the 184 single main RAs enter the kidney through the hilum. Regarding the aRAs, Table 2 summarizing the distribution of aRAs position to the kidneys:

Table 2: distribution of aberrant renal arteries Entrance to the kidneys.

\begin{tabular}{|c|c|c|}
\hline Position to the right kidney & n. & $\mathbf{( \% )}$ \\
\hline Lower pole & 23 & 58.9 \\
\hline Upper pole & 6 & 15.3 \\
\hline Hilum & 9 & 23 \\
\hline Upper pole and hilum & 1 & 2.5 \\
\hline Total & 39 & 100 \\
\hline Position to the left kidney & & \\
\hline Lower pole & 36 & 55.3 \\
\hline Upper pole & 19 & 29.2 \\
\hline Hilum & 9 & 13.8 \\
\hline Upper pole and hilum & 1 & 1.5 \\
\hline Total & 65 & 100 \\
\hline
\end{tabular}

No stable course observed in aRA as out of 59 cases with aRAs in the left side, a group of variations in the course of aRAs was seen in this study:

1) The left aRA originating from the aorta (just above main RA) and crossing the main RA to supply lower pole of the kidney instead of the upper pole that's demonstrated as nine cases (15.2\%) (Figure 2).
2) Regarding the right side, only one case (2.6 $\%)$ out of the 38 cases with aRA presented the crossing pattern; in which the aRA originating from the aorta and above the main RA then crossing it and enter the kidney hilum.

3) Two cases $(3.3 \%)$ demonstrating the aRA originating from the same trunk of the inferior mesenteric artery (Figure 3)
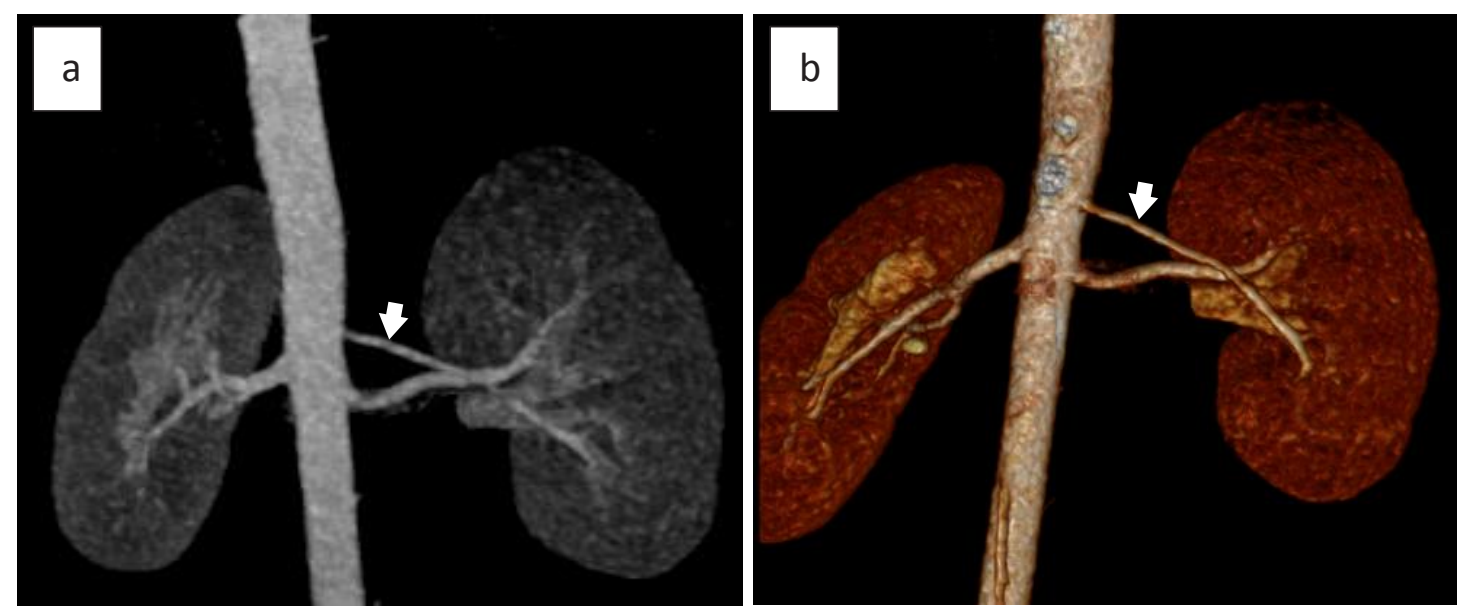

Figure 2: (a) and (b) showing the aRA (white arrow) arising just up to the main RAand crossing it to supply the lower pole of the left kidney. 


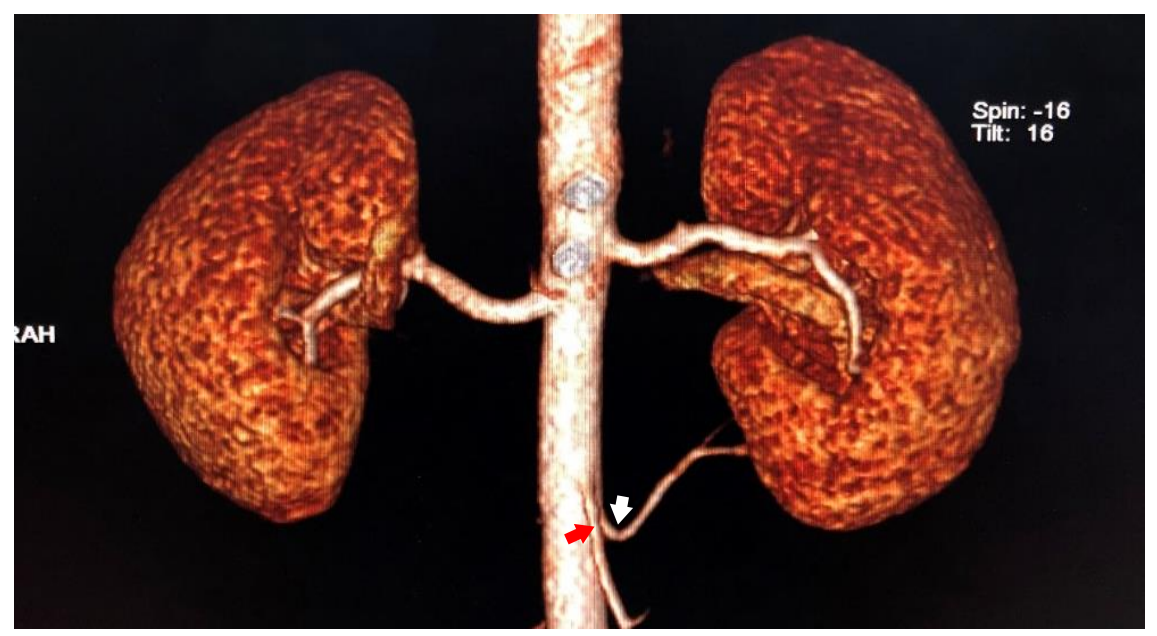

Figure 3: The left aRA (white arrow) arising from aorta at the same trunk of inferior mesenteric artery (red arrow).

Regarding the eRA, in the right side, one tiny artery demonstrated in two $(7.4 \%)$ cases that were branching into two branches, first one reaching the pericardial area and the second one reaching the right diaphragm area supplying the right dome of the diaphragm. In the left side, a one (5.2\%) tiny eRA was reaching the left diaphragmatic curs.
Relationship between the aberrant renal artery and the diameter of the main renal artery.

Table 3 summarizing the diameter of main RA in case the presence of aRAs or not. However, on the right side, the diameter of the main RA in kidney presenting the aRA was significantly less than that of the kidney with single main $\mathrm{RA}$ ( $\mathrm{t}$-test, $\mathrm{P}=0.006$ ). Regarding the left side, the diameter of the main RA in kidney presenting the aRA was significantly less than that of the kidney with single main $\mathrm{RA}$ (t-test, $\mathrm{P}=0.01$ ).

Table 3: Sum marizing the diam eter of main RA in case presence of aRAs or not.

\begin{tabular}{|c|c|c|c|c|}
\hline Symm etry & No. & Mean diameter of main RA ( $\mathrm{m} \mathrm{m}$ ) & SD & $\mathbf{P}$ \\
\hline \multicolumn{4}{|l|}{ Right side } & \multirow{4}{*}{0.006} \\
\hline aRAs present & 38 & 5.60 & 1.12571 & \\
\hline aRAs not present & 146 & 6.12 & 0.99664 & \\
\hline Total & 184 & 11.73 & 2.12235 & \\
\hline \multicolumn{4}{|l|}{ Left side } & \multirow{4}{*}{0.01} \\
\hline aRAs present & 59 & 5.87 & 1.00817 & \\
\hline aRAs not present & 125 & 6.27 & 1.01004 & \\
\hline Total & 184 & 12.1514 & 2.01821 & \\
\hline
\end{tabular}

No.: number of cases, aRAs: aberrant renal arteries, $(\mathrm{mm})$ : millimeter, SD: standard deviation. 


\section{Discussion}

Variations in the renal arteries are common, this explained by the development of the mesonephric arteries that's degenerates leaving only one mesonephric artery. Failure in degeneration of other mesonephric arteries results in an aberrant renal artery. This study is the first work in Iraq deals with aberrant renal arterial variation in details covering 184 kidneys donors' cases with medicolegal acceptance in Erbil city. This study showed the prevalence of aRAs of $26.3 \%$ compared with $20.2 \%$ in another angiographic study in Iraq [12] and within established ranges (20-30\%) by most authors and textbooks [2\&5]. The frequency of multiple renal arteries varies widely with ethnicity [13]. According to Gulas et al., the range of the frequency of multiple renal arteries depending on ethnicity is between 4\% (Malaysians) and $61.5 \%$ (Indians) [14]. In the Polish population, the variability of multiple renal arteries seems to be between $11.2 \%$ and $38.3 \%$ [15]. This difference can be explained by the wide range of the prevalence of aRAs as showed by Satyapal et al (9-76\%, average 28\%) [13].

In this study, the entrance of aRAs to the kidneys was also studied. In the right side, $58.9 \%$ of the aRAs enters the lower pole of the kidneys, $23 \%$ of aRAs enters through the hilum, $15.3 \%$ enters the upper pole of the kidney. Regarding the left side, $55.3 \%$ of the aRAs directed toward the upper pole, and $29.2 \%$ passing to the upper pole, and $13.8 \%$ of the aRAsenters through the hilum. These findings matched with Alghizzi findings but the percentage lower than current study; Alghizzi reported the most of the aRAs was an inferior polar artery in $63.6 \%$ [12]. But present study findings disagreed with findings reported by Mustafa et al in which the results showed most of aRAs are pass through the hilum $53.62 \%$, about $32.85 \%$ of the arteries enter the upper pole, and only $13.53 \%$ of the arteries enterthe lower pole of the kidney [20]. Rarely, in the right side, only $2.5 \%$ aRA gives branch to upper pole and hilum of the kidney. Regarding the left side, only $1.5 \%$ of aRA gives branch to upper pole and hilum of the kidney.

There were variations in the course of aRA as interestingly, the leftaRA originating from the aorta just above main RA and crossing it to supply lower pole of the kidney instead of the upper pole that's demonstrated as cases $15.2 \%$. Regarding the right side, only $2.6 \%$ of the cases with aRA presented the crossing pattern; in which the aRA originating from the aorta and above the main
RA then crossing it and enter the kidney hilum. About $3.3 \%$ of the cases demonstrating the aRA originating from the same trunk of the inferior mesenteric artery (Figure 3).

Conclusion: This study concluded that:

1. The prevalence of aberrant renal arteries is $26.3 \%$ of Iraqi population.

2. Regarding the incidence side, it's more common in the left side (32.1\%) than the right side $(20.7 \%)$.

3. Presence of aberrant renal artery will affect the diameter of main renal artery; in which the diameter of the main RA in kidney presenting the aRA was significantly less than that of the kidney with single main RA.

4. No stable course observed in aRA in which the aRA originating from the aorta just above main RA and crossing the main RA to supply lower pole of the kidney instead of the upper pole.

5. Regarding the early branch renal artery, in the right side a one tiny eRA branching into two branches, one tiny artery reaching the pericardial area; and another artery that's reaching the right diaphragm area Source of Funding- Self

Ethical Clearance - Not required

Conflict of Interest: None.

\section{References}

[1]. Graves FT. The aberrant renal artery. J Anat. 1956 Oct;90(Pt 4):553-558.1.

[2]. Standring S. Kidney and ureter. In: Guzzo, TJ, Torigian DA, editors. Gray's Anatomy International Edition: The Anatomical Basis of Clinical Practice. 41th ed. New York: Elsevier Limited; 2016.

[3]. Nagpal H, Rajni D, Gupta M, Adhikari SR, Mishra R. ACCESSORY RENAL ARTERY AND ACCESSORY RENAL VEIN IN RIGHT KIDNEY - A CASE REPORT. Int J Sci Res [Internet]. 2018 Aug 3 [cited 2019 Mar 5];7(7).

[4]. Sinnatamby CS, Last RJ, editors. Last's anatomy: regional and applied. 12th ed. Edinburgh ; New York: Churchill Livingstone/ Elsevier; 2011. 548 p.

[5]. Moore KL, Dalley AF, Agur AMR. Clinically 
Oriented Anatomy. 8th ed. Philadelphia: Wolters Kluwer; 2018. 1171 p.

[6]. Gillaspie C, Miller LI, Baskin M. Anomalous renal vessels and their surgical significance. Anat Rec. 1916 Oct;11(3):77-86.

[7]. Gardner S. An Accessory Left Renal Artery: A Case Report. Austin J Anat. 2015;2(3):1041.

[8]. Keibel F, Mall FP. Manual of Human Embryology; Vol. 1. Creative Media Partners, LLC; 2018. 568 p.

[9]. Schoenwolf GC, Bleyl SB, Brauer PR, FrancisWest PH. Larsen's human embryology. Fifth edition. Philadelphia, PA: Churchill Livingstone; 2015. 554 p.

[12]. ALghizzi HJK. The variation of renal artery anatomy in the Iraqi population: An angiographic study of 358 middle Euphrates Iraqi patients attending the catheterization laboratory in Hilla province. Karbala J Med. 2011;4(1,2):933-8.

[13]. Satyapal KS, Haffejee AA, Singh B, Ramsaroop L, Robbs JV, Kalideen JM. Additional renal arteries incidence and morphometry. Surg
Radiol Anat. 2001 Apr;23(1):33-8.

[14]. Gulas E, Wysiadecki G, Szymański J, Majos A, Stefańczyk L, Topol M, et al. Morphological and clinical aspects of the occurrence of accessory (multiple) renal arteries. Arch Med Sci. 2018;14(2):442-53.

[15]. Wróbel G, Spałek M, Kuchinka J, Kuder T. Four left renal arteries - a rare variant of kidney arterial supply. Folia Morphol [Internet]. 2015 Sep 3 [cited 2019 Aug 9]

[16]. Zağyapan R. A retrospective study on multiple renal arteries in Turkish population. Anat Int J Exp Clin Anat. 2009;3:35-9.

[17]. Kaneko N, Kobayashi Y, Okada Y. Anatomic variations of the renal vessels pertinent to transperitoneal vascular control in the management of trauma. Surgery. 2008 May;143(5):616-22.

[18]. Khamanarong K, Prachaney P, Utraravichien A, Tong-Un T, Sripaoraya K. Anatomy of renal arterial supply. Clin Anat. 2004;17(4):334-6. 